\title{
FATORES DE RISCO PARA DIARRÉIA PERSISTENTE EM LACTENTES
}

\author{
Maria das Graças Moura LINS, Maria Eugênia Farias Almeida MOTTA e Giselia Alves Pontes da SILVA
}

RESUMO - Racional - A diarréia persistente é uma doença multicausal. A análise do risco para o prolongamento do quadro diarréico envolve variáveis ambientais, biológicas e do manejo clínico. Objetivo - Identificar fatores de risco para a diarréia persistente em crianças hospitalizadas na fase aguda do quadro diarréico. Pacientes e Métodos - O estudo foi do tipo caso-controle. A amostra consistiu de 216 crianças menores de 24 meses hospitalizadas por diarréia de início abrupto, no Instituto Materno-Infantil de Pernambuco, Recife, PE. O grupo de casos incluiu as crianças com diarréia persistente e o de controles aquelas com diarréia aguda. Foram analisadas variáveis socioeconômicas, biológicas, de morbidade anterior, clínicas e do manejo terapêutico prévio à admissão. Utilizou-se o odds ratio não ajustado e ajustado, com seus respectivos intervalos de confiança de 95\%, observando-se o nível de significância de 5\%. A análise multivariada foi feita através de regressão logística. Resultados - O risco de persistência da diarréia foi maior nas crianças com: disenteria, febre no início do quadro, dieta suspensa e uso de antibiótico à admissão hospitalar. O risco de diarréia persistente foi cerca de três vezes maior para crianças sem geladeira no domicílio e que apresentavam hiperemia perianal ao exame físico na admissão hospitalar, sendo estas as variáveis que apresentaram significância estatística após o ajuste para fatores de confusão. Conclusões - A melhoria das condições ambientais e o manejo adequado e individualizado da criança hospitalizada por diarréia pode contribuir para a redução da morbidade da doença.

DESCRITORES - Diarréia infantil. Fatores de risco. Hiperemia.

\section{INTRODUÇÃO}

A epidemiologia da diarréia persistente (DP) ainda é pouco conhecida, especialmente porque os dados sobre incidência são variáveis de uma região para outra, fato este que pode estar relacionado aos diferentes fatores causais que atuam e predominam em cada área. Estima-se que entre 3\% e 20\% dos episódios diarréicos infecciosos que acometem menores de 5 anos tornam-se persistentes ${ }^{(4,21,30)}$. Taxas elevadas encontradas nos países em desenvolvimento contrastam com os baixos índices nos países desenvolvidos e, como a DP é mais freqüente em crianças que tiveram episódios anteriores de diarréia, é possível que o problema concentre-se em pequeno número delas ${ }^{(4,22)}$.

A identificação de fatores de risco diretamente relacionados à DP é fundamental para a compreensão de sua gênese e prevenção. Baixa idade, desnutrição, comprometimento da resposta imune celular, infecções prévias, diarréia por E. coli enteroaderente, E.coli enteropatogênica clássica (EPEC) e Shigella, manejo inadequado e drogas adminis- tradas na fase aguda contribuem para o curso prolongado da diarréia $^{(15,20,21)}$. Algumas características da fase inicial do episódio diarréico, como diarréia de alto débito e presença de leucócitos, pus ou sangue nas fezes, predizem sua possível persistência ${ }^{(5,28)}$.

A diarréia persistente pode apresentar-se de forma leve, moderada ou grave. Na leve, a perda hidroeletrolítica é discreta, mas há dificuldade para ganhar peso, mesmo quando a ingestão de alimentos mantém-se normal. Os quadros moderados caracterizam-se por labilidade hidroeletrolítica, maior freqüência de evacuações e má absorção intestinal, que são controlados por dieta adequada e reidratação oral. Nas formas graves, ocorrem labilidade eletrolítica e comprometimento do estado nutricional importantes, com morbidade e letalidade elevadas ${ }^{(1,6,9)}$. Estes casos, apesar de acometerem menor número de crianças, necessitam, invariavelmente, de tratamento hospitalar e contribuem sobremaneira para a duração prolongada do internamento, com altos custos e taxas de mortalidade elevadas nos serviços ${ }^{(2,23)}$. 
Considerando que a contribuição das variáveis envolvidas na gênese da DP pode diferir de acordo com as características da população analisada, este estudo teve como objetivo identificar os fatores de risco associados ao curso persistente da diarréia em lactentes atendidos em um serviço de saúde conveniado ao Sistema Único de Saúde (SUS), na cidade do Recife, PE.

\section{CASUÍSTICA E MÉTODOS}

O estudo foi do tipo caso-controle e desenvolveu-se no Hospital Geral de Pediatria do Instituto Materno-Infantil de Pernambuco. Dele participaram crianças menores de 24 meses com doença diarréica de início abrupto, hospitalizadas por apresentarem sinais de desidratação, no período de junho de 1993 a março de 1994 e cujas mães se comprometeram a comparecer às consultas subseqüentes ao internamento. As crianças que preencheram os critérios de inclusão e exclusão foram admitidas ao estudo (casos incidentes).

Foram agendadas consultas ambulatoriais até a limitação do episódio diarréico para as crianças que receberam alta hospitalar ainda na fase aguda da diarréia, a fim de conhecer a sua evolução. Este período de acompanhamento prospectivo permitiu definir o episódio diarréico como agudo - diarréia de início abrupto que se limitou até o 14 ia - ou persistente - quadro com duração maior do que 14 dias. O grupo de casos incluiu as crianças com diarréia persistente e o de controles, aquelas com diarréia aguda. Os critérios de exclusão foram doenças sistêmicas graves associadas à diarréia e história clínica compatível com doenças crônicas do trato gastrointestinal.

O cálculo do tamanho da amostra foi feito através do software EPI-INFO, versão 6.0. A partir das 50 primeiras crianças admitidas no estudo, estabeleceu-se a proporção de expostos aos fatores de risco representativos de cada bloco de variáveis (socioeconômicas e demográficas, biológicas, de morbidade, características clínicas e de manejo do episódio diarréico atual). Com estes dados, considerando-se nível de significância de 0,05 e "power" de $80 \%$, calculou-se a amostra necessária para cada uma das variáveis relevantes, na proporção de 1 caso para 1 controle, e o maior deles resultou em um tamanho estimado de 90 crianças em cada grupo, mas o período de coleta de dados permitiu a inclusão de maior número de pacientes (106 casos e 106 controles).

Para a coleta de dados, utilizou-se formulário estruturado construído a partir dos questionários desenvolvidos pelos Programas de Controle das Doenças Diarréicas (PCDD)(3) da Organização Mundial de Saúde, contendo dados da história clínica e exame físico. As mães eram esclarecidas sobre as finalidades e os procedimentos previstos para a pesquisa e, em seguida, solicitadas à concordância para a participação de seu filho e assinado o termo de consentimento livre e esclarecido.
O projeto foi aprovado pelo Comitê de Ética em Pesquisa do Centro de Ciências da Saúde da Universidade Federal de Pernambuco e pelo Comitê de Ética em Pesquisa do Instituto Materno-Infantil de Pernambuco.

A diarréia foi caracterizada como a passagem de três ou mais evacuações líquidas em período de 24 horas. Definiu-se o episódio diarréico como aquele iniciado nas primeiras 24 horas em que se caracterizou a diarréia e terminado no último dia desta. A definição das características clínicas e de manejo do episódio resultou da avaliação da criança no momento da admissão ao hospital, através dos dados da anamnese e do exame físico. O estado de desidratação foi definido como leve, moderado e grave, de acordo com a classificação do Ministério da Saúde ${ }^{(7)}$. Considerou-se disenteria a presença de sangue visível nas fezes. O estado nutricional foi classificado segundo o índice peso/comprimento, utilizando-se a curva de referência do National Center for Health Statistics (NCHS) e apresentado sob a forma de percentis. Os padrões técnicos da aferição antropométrica seguiram as orientações de GIBSON ${ }^{(14)}$.

A análise estatística foi realizada com os softwares EPI-INFO, versão 6.0 e SPSS for Windows, versão 8.0. As variáveis foram incluídas na análise como categóricas. A análise bivariada foi feita entre a classificação da diarréia (persistente e aguda) e cada uma das variáveis socioeconômicas, biológicas, de morbidade, clínicas e do manejo do quadro diarréico. Utilizou-se o "odds ratio" (OR) não ajustado, com seu respectivo intervalo de confiança de 95\% (IC 95\%), e o nível de significância de 5\% para aceitação da associação estatística. O OR foi calculado em relação à categoria basal (aquela com menor risco para a persistência da diarréia), tanto para o OR nãoajustado quanto para o OR ajustado.

A análise multivariada foi feita através da regressão logística seqüencial (método ENTER), para controlar possíveis fatores de confusão. De acordo com recomendações de VICTORA et al. ${ }^{(32)} \mathrm{e}$ FUCHS et al. ${ }^{(13)}$, a decisão sobre quais fatores de risco incluir no modelo baseia-se em uma estrutura conceitual, descrevendo as relações hierárquicas entre eles. Fundamentando-se nesta afirmativa, as variáveis foram incluídas no modelo de forma hierarquizada, sendo que todas as variáveis de cada nível entraram na análise ajustada. Aquelas que continuavam significativas ao nível de $20 \%$ eram mantidas no modelo e participavam do ajuste do próximo bloco; uma vez selecionada, a variável permanecia nos modelos subseqüentes, mesmo que perdesse a significância com a inclusão de variáveis hierarquicamente inferiores. No modelo estruturado para esta pesquisa, introduziram-se no primeiro bloco as variáveis socioeconômicas. No segundo, acrescentaram-se as biológicas (peso ao nascer, período de aleitamento materno, idade e índice peso/comprimento). No terceiro, incluíram-se as variáveis indicadoras de morbidade (diarréia nos últimos 3 meses e hospitalização anterior). No último bloco, introduziram-se 
as variáveis clínicas (tipo de diarréia, febre, vômito, estado de hidratação e hiperemia perianal) e de manejo prévio à admissão hospitalar (pausa alimentar $>24$ horas e prescrição de antibiótico).

\section{RESULTADOS}

Foram estudadas 212 crianças, das quais $129(60,8 \%)$ eram do sexo masculino e $83(39,2 \%)$ do feminino. Quanto à faixa etária, $126(59,4 \%)$ eram menores de 6 meses e $86(40,6 \%)$ tinham 6 ou mais meses. A idade média foi de 5,3 meses ( $3 \mathrm{~m}-24 \mathrm{~m})$. Em relação ao aleitamento materno, 44,3\% (94/212) foram amamentadas durante o primeiro mês de vida, 21,7\% (46/212) não receberam aleitamento materno e, no momento da admissão, nenhuma criança recebia leite materno.

Quanto ao tempo de doença diarréica no momento da admissão hospitalar, 39,2\% (83/212) tinham menos de 7 dias de doença, 27,8\% (59/212) entre 7 e 14 dias e 33,0\% (70/212) mais de 14 dias.
Nas Tabelas 1, 2 encontram-se as distribuições de freqüências e a estimativa do risco das variáveis socioeconômicas e biológicas, de acordo com a classificação do episódio diarréico.

$\mathrm{O}$ risco de persistência da diarréia foi duas vezes maior em crianças com disenteria em relação àquelas com diarréia aquosa, com associação significativa na análise bivariada $(P<0,01)$. O risco de evoluir para diarréia persistente foi maior entre as crianças que na fase inicial do episódio diarréico apresentaram febre $(P<0,01)$ e naquelas que tiveram a alimentação suspensa por mais de 24 horas $(P=0,05)$. As crianças que usaram antibiótico para a diarréia antes da admissão no hospital tiveram risco quase 4 vezes maior de evoluírem para diarréia persistente e a diferença estatística foi significativa na análise bivariada $(P<0,001)$ (Tabela 3 ).

Após o ajuste para fatores de confusão, apenas duas variáveis explicaram a persistência da diarréia: presença de geladeira no

TABELA 1 - Classificação do episódio diarréico de acordo com variáveis socioeconômicas demográficas

\begin{tabular}{|c|c|c|c|}
\hline \multirow[b]{2}{*}{ Variáveis } & \multicolumn{2}{|c|}{ Diarréia } & \multirow[b]{2}{*}{$\begin{array}{l}\text { OR bruto } \\
\text { (IC } 95 \%)\end{array}$} \\
\hline & $\begin{array}{c}\text { Persistente } \\
\text { n (\%) }\end{array}$ & $\begin{array}{c}\text { Aguda } \\
\text { n (\%) }\end{array}$ & \\
\hline \multicolumn{4}{|l|}{ Renda } \\
\hline > 1 Salário mínimo & $51(48,1)$ & $45(44,1)$ & 1,00 \\
\hline$\leq 1$ Salário mínimo & $55(51,9)$ & $57(55,9)$ & $0,85(0,49-1,47)^{*}$ \\
\hline \multicolumn{4}{|l|}{ Água encanada } \\
\hline Sim, dentro do domicílio & $32(30,2)$ & $36(34,3)$ & 1,00 \\
\hline Sim, no quintal & $38(35,9)$ & $33(31,4)$ & $1,30(0,67-2,52)^{*}$ \\
\hline Não & $36(33,9)$ & $36(34,3)$ & $1,13(0,58-2,18)^{*}$ \\
\hline \multicolumn{4}{|l|}{ Tipo de sanitário } \\
\hline Com descarga & $30(28,3)$ & $26(24,8)$ & 1,00 \\
\hline Sem descarga & $76(71,7)$ & $79(75,2)$ & $0,83(0,45-1,54)^{*}$ \\
\hline \multicolumn{4}{|l|}{ Tipo de construção } \\
\hline Tijolo & $72(67,9)$ & $69(66,3)$ & 1,00 \\
\hline Outros & $34(32,1)$ & $35(33,7)$ & $0,93(0,52-1,66)^{*}$ \\
\hline \multicolumn{4}{|l|}{ Geladeira no domicílio } \\
\hline Sim & $20(18,9)$ & $32(30,5)$ & 1,00 \\
\hline Não & $86(81,1)$ & $73(69,5)$ & $1,88(0,99-3,57)^{4}$ \\
\hline \multicolumn{4}{|l|}{ Escolaridade materna } \\
\hline$>5$ anos & $20(18,9)$ & $15(14,6)$ & 1,00 \\
\hline 1-4 anos & $59(55,7)$ & $68(66,0)$ & $0,65(0,31-1,38)^{*}$ \\
\hline Nenhuma & $27(25,4)$ & $20(19,4)$ & $1,01(0,42-2,45)^{*}$ \\
\hline \multicolumn{4}{|l|}{ Tamanho da família } \\
\hline $2-3$ pessoas & $20(18,9)$ & $21(19,8)$ & 1,00 \\
\hline $4-5$ pessoas & $47(44,3)$ & $50(47,2)$ & $0,99(0,48-2,05)^{*}$ \\
\hline 6 pessoas & $39(36,8)$ & $35(33,0)$ & $1,17(0,55-2,51)^{*}$ \\
\hline
\end{tabular}

* $P>0,05 ;{ }^{\S} P<0,05 ;{ }^{`} P=0,05$ 
TABELA 2 - Classificação do episódio diarréico de acordo com variáveis biológicas

\begin{tabular}{|c|c|c|c|}
\hline \multirow[b]{2}{*}{ Variáveis } & \multicolumn{2}{|c|}{ Diarréia } & \multirow[b]{2}{*}{ OR bruto (IC 95\%) } \\
\hline & Persistente n (\%) & Aguda n (\%) & \\
\hline \multicolumn{4}{|l|}{ Grupo de idade } \\
\hline$\geq 6$ meses & $52(49,0)$ & $34(32,0)$ & 1,00 \\
\hline$<6$ meses & $54(51,0)$ & $72(68,0)$ & $0,49(0,28-0,86)^{\S}$ \\
\hline \multicolumn{4}{|l|}{ Peso ao nascer } \\
\hline$\geq 2.500 \mathrm{~g}$ & $80(85,1)$ & $70(74,5)$ & $1,00 \dagger$ \\
\hline$<2.500 \mathrm{~g}$ & $14(14,9)$ & $24(25,5)$ & $0,51(0,24-1,06)^{*}$ \\
\hline \multicolumn{4}{|c|}{ Duração do aleitamento materno } \\
\hline$>3$ meses & $10(9,4)$ & $8(7,6)$ & 1,00 \\
\hline $1-2$ meses & $71(67,0)$ & $72(68,6)$ & $0,79(0,29-2,11)^{*}$ \\
\hline Não amamentou & $25(23,6)$ & $25(23,8)$ & $0,80(0,27-2,36)^{*}$ \\
\hline \multicolumn{4}{|l|}{ Hospitalização anterior } \\
\hline Não & $33(31,1)$ & $56(52,8)$ & 1,00 \\
\hline Sim & $73(68,9)$ & $50(47,2)$ & $2,48(1,41-4,34)^{\S}$ \\
\hline \multicolumn{4}{|c|}{ Índice peso/comprimento } \\
\hline$\geq \mathrm{P}_{10}$ & $44(37,7)$ & $47(44,8)$ & 1,00 \\
\hline $\mathrm{P}_{3}-\mathrm{P}_{10}$ & $20(18,9)$ & $12(11,4)$ & $1,78(0,78-4,06)^{*}$ \\
\hline$<\mathrm{P} 3$ & $42(39,6)$ & $46(43,8)$ & $0,98(0,54-1,75)^{*}$ \\
\hline \multicolumn{4}{|c|}{ Diarréia nos últimos 3 meses } \\
\hline Não & $71(67,0)$ & $80(76,2)$ & 1,00 \\
\hline Sim & $35(33,0)$ & $25(23,8)$ & $1,58(0,86-2,89)^{*}$ \\
\hline
\end{tabular}

TABELA 3 - Classificação do episódio diarréico de acordo com variáveis de morbidade e manejo clínico

\begin{tabular}{|c|c|c|c|}
\hline \multirow[b]{2}{*}{ Variáveis } & \multicolumn{2}{|c|}{ Diarréia } & \multirow[b]{2}{*}{ OR bruto (IC 95\%) } \\
\hline & Persistente n (\%) & Aguda n (\%) & \\
\hline \multicolumn{4}{|l|}{ Tipo de diarréia } \\
\hline Aquosa & $46(43,4)$ & $65(61,3)$ & 1,00 \\
\hline Disentérica & $60(56,6)$ & $41(38,7)$ & $2,07(1,20-3,58)^{\pi}$ \\
\hline \multicolumn{4}{|l|}{ Vômito } \\
\hline Não & $25(23,6)$ & $32(30,5)$ & 1,00 \\
\hline $\operatorname{Sim}$ & $81(76,4)$ & $73(69,5)$ & $1,42(0,77-2,62) \dagger$ \\
\hline \multicolumn{4}{|l|}{ Febre } \\
\hline Não & $17(16,0)$ & $33(31,4)$ & 1,00 \\
\hline $\operatorname{Sim}$ & $89(84,0)$ & $72(68,6)$ & $2,40(1,24-4,65)^{\bullet}$ \\
\hline \multicolumn{4}{|l|}{ Estado de hidratação } \\
\hline Hidratado & $10(9,4)$ & $9(8,5)$ & 1,00 \\
\hline Desidratado leve/moderado & $82(77,4)$ & $85(80,2)$ & $0,87(0,34-2,25) \dagger$ \\
\hline Desidratado grave & $14(13,2)$ & $12(11,3)$ & $1,05(0,32-3,44) \dagger$ \\
\hline \multicolumn{4}{|l|}{ Hiperemia perianal } \\
\hline Não & $34(32,0)$ & $65(62,5)$ & 1,00 \\
\hline Sim & $72(68,0)$ & $39(37,5)$ & $3,53(2,00-6,23)^{*}$ \\
\hline \multicolumn{4}{|l|}{ Pausa alimentar $>24 \mathrm{~h}$} \\
\hline Não & $59(55,7)$ & $73(68,9)$ & 1,00 \\
\hline $\operatorname{Sim}$ & $47(44,3)$ & $33(31,1)$ & $1,76(1,01-3,09)^{\S}$ \\
\hline \multicolumn{4}{|c|}{ Prescrição de antibiótico para diarréia } \\
\hline Não & $23(22,5)$ & $52(52,0)$ & 1,00 \\
\hline Sim & $79(77,5)$ & $48(48,0)$ & $3,72(2,03-6,83)^{*}$ \\
\hline
\end{tabular}

Níveis de significância: ${ }^{\circledR} P<0,01 ;{ }^{\S} P<0,05 ; * P<0,001 ; \dagger P>0,05 ; \bullet P=0,01$ 
domicílio e hiperemia perianal (Tabela 4). O risco para diarréia persistente foi cerca de 3 vezes maior para crianças sem geladeira no domicílio e que apresentavam hiperemia perianal ao exame físico na admissão hospitalar.

\section{DISCUSSÃO}

O conhecimento da epidemiologia da doença diarréica é fundamental para que medidas preventivas e terapêuticas possam ser implementadas, com conseqüente redução na morbiletalidade. Para a decisão das medidas mais adequadas a cada caso, é importante analisar o comportamento da doença desde sua expressão na comunidade, até os casos que chegam ao hospital, certamente os mais graves.

A identificação dos fatores de risco associados à ocorrência do episódio diarréico, à evolução clínica e à avaliação dos fatores prognósticos relacionados ao agravamento do quadro, permitem abordagem racional da doença, contribuindo para a redução do número de hospitalizações e da taxa de óbitos. O médico pode intervir nesta cadeia de eventos em vários momentos: implementando medidas educativas (promoção da saúde, higiene pessoal, alimentar e doméstica) ${ }^{(10,25)}$, incentivando o aleitamento materno por tempo prolongado $^{(10,21,25)}$, promovendo o manejo adequado na fase aguda do processo diarréico ${ }^{(4,12,16,20)}$ e prevenindo a instalação/agravamento dos déficits nutricionais ${ }^{(9,16,33)}$.

Embora a terapia de reidratação oral (TRO) tenha sido implantada no nosso meio desde a década de 1980, ainda se observa, infelizmente, que o manejo na fase aguda do episódio diarréico ainda é inadequado em grande parte do país, de modo especial na região nordeste ${ }^{(22)}$. As informações obtidas neste estudo, realizado no Instituto Materno-Infantil de Pernambuco, são úteis para o melhor entendimento da doença diarréica em crianças atendidas em unidade hospitalar conveniada com o SUS em nosso meio.

Vários autores têm demonstrado a associação de variáveis socioeconômicas e demográficas, tanto com a ocorrência do episódio diarréico, quanto com o curso clínico, sendo a doença mais freqüente e mais grave entre as crianças pertencentes às famílias de menor renda ${ }^{(1,8,19,24)}$. O nível de renda familiar tem sido utilizado como indicador de bem-estar social, pois permite o acesso a bens de consumo que proporcionam boa qualidade de vida como moradia, saneamento e educação ${ }^{(15,30)}$.

Neste estudo, essas associações não foram observadas, provavelmente porque as crianças eram provenientes de famílias com

TABELA 4 - Regressão logística seqüencial de fatores de risco para diarréia persistente

\begin{tabular}{|c|c|c|c|c|}
\hline \multirow[b]{2}{*}{ Variáveis } & \multicolumn{2}{|c|}{ Diarréia } & \multirow{2}{*}{$\begin{array}{l}\text { OR bruto } \\
\text { (IC 95\%) }\end{array}$} & \multirow{2}{*}{$\begin{array}{l}\text { OR ajustado } \\
\text { (IC 95\%) }\end{array}$} \\
\hline & Persistente n (\%) & Aguda n (\%) & & \\
\hline \multicolumn{5}{|l|}{ Água encanada } \\
\hline Sim, dentro do domicílio & $32(30,2)$ & $36(34,3)$ & $1,00^{*}$ & 1,00 \\
\hline Sim, no quintal & $38(35,9)$ & $33(31,4)$ & $1,30(0,67-2,52)^{\bullet}$ & $1,94(0,62-6,01)^{\bullet}$ \\
\hline Não & $36(33,9)$ & $36(34,3)$ & $1,13(0,58-2,18)^{\bullet}$ & $2,63(0,88-7,85)^{\bullet}$ \\
\hline \multicolumn{5}{|l|}{ Tipo de sanitário } \\
\hline Com descarga & $30(28,3)$ & $26(24,8)$ & $1,00^{*}$ & 1,00 \\
\hline Sem descarga & $76(71,7)$ & $79(75,2)$ & $0,83(0,45-1,54)^{\bullet}$ & $0,43(0,15-1,24)^{\bullet}$ \\
\hline \multicolumn{5}{|l|}{ Geladeira no domicílio } \\
\hline Sim & $20(18,9)$ & $32(30,5)$ & $1,00 *$ & 1,00 \\
\hline Não & $86(81,1)$ & $73(69,5)$ & $1,88(0,99-3,57) \dagger$ & $2,75(1,11-6,82)^{\S}$ \\
\hline \multicolumn{5}{|l|}{ Peso ao nascer } \\
\hline$\geq 2500 \mathrm{~g}$ & $80(85,1)$ & $70(74,5)$ & $1,00 \dagger$ & 1,00 \\
\hline$<2500 \mathrm{~g}$ & $14(14,9)$ & $24(25,5)$ & $0,51(0,24-1,06)^{\bullet}$ & $0,65(0,26-1,61)^{\bullet}$ \\
\hline \multicolumn{5}{|l|}{ Grupo de idade } \\
\hline$\geq 6$ meses & $52(49,0)$ & $34(32,0)$ & $1,00 \dagger$ & 1,00 \\
\hline$<6$ meses & $54(51,0)$ & $72(68,0)$ & $0,49(0,28-0,86)^{\$}$ & $0,91(0,43-1,94)^{\bullet}$ \\
\hline \multicolumn{5}{|l|}{ Hospitalização anterior } \\
\hline Não & $33(31,1)$ & $56(52,8)$ & $1,00 \ddagger$ & 1,00 \\
\hline Sim & $73(68,9)$ & $50(47,2)$ & $2,48(1,41-4,34)^{\S}$ & $1,81(0,87-3,79)^{\bullet}$ \\
\hline \multicolumn{5}{|l|}{ Hiperemia perianal } \\
\hline Não & $34(32,0)$ & $65(62,5)$ & $1,00^{\Psi}$ & 1,00 \\
\hline Sim & $72(68,0)$ & $39(37,5)$ & $3,53(2,00-6,23)^{\Phi}$ & $2,74(1,30-5,76)^{\pi}$ \\
\hline
\end{tabular}

Variáveis do modelo: *bloco 1 ; †bloco 2 ; $₫$ bloco $3 ;{ }^{\Psi}$ bloco 4

Níveis de significância: ${ }^{\S} P<0,05 ; \Phi P<0,001 ;{ }^{\uparrow} P<0,01 ; \bullet P>0,05 ; \dagger P=0,05 ;{ }^{\circledR} P=0,01$ 
condições socioeconômicas semelhantes. A única variável socioeconômica que apresentou significância estatística foi a presença de geladeira no domicílio, incluída no modelo de regressão logística, por ser um modo indireto de avaliação do poder aquisitivo da família e por sugerir que as famílias possuidoras deste bem estão mais preocupadas em garantir a qualidade de alimentos consumidos. As famílias não possuidoras de geladeira estariam em situação de maior vulnerabilidade no tocante à aquisição de doenças passíveis de transmissão através da contaminação de água e alimentos.

Diversos autores têm demonstrado que o nível de escolaridade da mãe guarda relação com a qualidade dos cuidados oferecidos aos filhos ${ }^{(22,29)}$ Estudo de revisão sobre doença diarréica em menores de 5 anos nas Américas evidenciou que cada ano adicional de escolaridade da mãe foi capaz de reduzir em $7 \%$ a incidência da doença em seus filhos ${ }^{(22)}$. As mães com maior número de anos de estudo demonstram ter melhores condições de compreender as informações dadas pelos profissionais da área da saúde e de aplicar o conhecimento adquirido na melhoria do cuidado infan$\mathrm{til}^{(31)}$. Elas enfrentam de maneira mais efetiva os problemas associados com a pobreza, compreendem e retêm informações sobre amamentação, noções de higiene e uso adequado de sais de reidratação oral, entre outras orientações. Observa-se que seus filhos apresentam melhor nível de cobertura vacinal, menor índice de doenças, de internações e de deficiências nutricionais. Vários estudos têm demonstrado a importância deste indicador, independentemente de outras variáveis sociais ${ }^{(26,29)}$.

Apesar de ter sido observada maior proporção de mães que não freqüentaram a escola entre as crianças com diarréia persistente, a diferença observada não foi estatisticamente significante, não permitindo analisar, nesta amostra, o impacto desta variável.

Demonstrou-se que crianças desnutridas, desmamadas precocemente e que tenham tido episódios recentes de diarréia ou qualquer outra morbidade que comprometa o estado nutricional, apresentam risco maior para diarréia persistente ${ }^{(3,8,22,27,33)}$. Ao uso do leite materno se atribui impacto significativo na redução dos percentuais de morbidade e mortalidade por diarréia ${ }^{(1,11,16)}$. Esta relação pode ser explicada pelas propriedades antimicrobianas e imunológicas inerentes a sua composição, devendo também ser considerada a sua capacidade de garantir o adequado estado nutricional do lactente, assim como a isenção de contaminação quando o seu uso é exclusivo $^{(16)}$. O desmame precoce e a introdução inadequada dos alimentos de transição tanto no que se refere a oferta calórica, quanto do preparo dos alimentos, favorecem a instalação de déficits nutricionais e a exposição aos enteropatógenos potenciais ${ }^{(25,26)}$. Essa associação tem sido freqüentemente demonstrada por diferentes pesquisadores em várias regiões do mundo ${ }^{(5,10)}$. Estudos prospectivos mostram associação da desnutrição com a duração do episódio diarréico. O risco aumentado para curso persistente da diarréia pode estar relacionado a vários fatores, pois a desnutrição, por si mesma, causa alterações importantes nas defesas do hospedeiro, como a redução da acidez gástrica, da motilidade intestinal, da síntese de anticorpos e da imunidade celular ${ }^{(16,18,33)}$.

Apesar das evidências observadas na literatura, não foram notadas diferenças com significância estatística entre o estado nutricional, o tempo de aleitamento materno, a ocorrência de episódios diarréicos nos 3 meses anteriores à doença atual e a evolução para diarréia persistente.

A presença de febre e sangue nas fezes, a suspensão da alimentação por mais de 24 horas e o uso de antibióticos estiveram associados ao prolongamento do episódio diarréico na análise não ajustada. Supõe-se que a diarréia que evolui com febre e sangue nas fezes esteja associada a enteropatógeno de maior virulência e, por isto, é menor a chance de o hospedeiro clarear a infecção em tempo mais curto. Por outro lado, o sangue nas fezes também pode refletir processo inflamatório associado à alergia alimentar, como conseqüência da maior absorção de macromoléculas protéicas por uma mucosa intestinal que tem sua permeabilidade aumentada devido ao insulto infeccioso ${ }^{(9,17)}$.

A suspensão da alimentação na fase aguda do episódio diarréico é um dos fatores de risco para a sua persistência e aumento do risco de morte por diarréia. A suspensão da dieta contribui diretamente para o agravamento do estado nutricional ${ }^{(14,23)}$, pois dificulta o reparo da mucosa intestinal, enquanto o uso de antimicrobianos altera a microbiota intestinal. Estas condutas associadas contribuem para o prolongamento da infecção, agravando a evolução do episódio diarréico ${ }^{(5,23)}$

Embora a presença de intolerância alimentar não tenha sido investigada por exames laboratoriais, a presença de hiperemia perianal sugere eliminação de fezes ácidas, provavelmente devido à intolerância secundária aos açúcares dietéticos, decorrente de lesão da mucosa do intestino delgado proximal, evento citado como um dos possíveis mecanismos perpetuadores do episódio diarréico ${ }^{(9)}$. Este achado pode ser considerado como fator prognóstico negativo quando identificado no momento da hospitalização, pois as crianças que apresentavam hiperemia perianal tiveram risco cerca de 3 vezes maior de evoluir para diarréia persistente. É interessante observar que esta foi a única variável clínica que manteve associação estatística na análise multivariada, o que sinaliza para a influência das variáveis clínicas no desfecho do episódio diarréico.

\section{CONCLUSÕES}

Pode-se concluir, com base nesta casuística, a importância de dois níveis de atuação clínica: a adoção de medidas preventivas que possam interferir na qualidade do ambiente, minimizando o impacto das condições socioeconômicas hostis que contribuam para a insta- 
lação do episódio diarréico, e o manejo adequado e individualizado da criança com diarréia aguda, evitando medidas que possam per- petuar o episódio diarréico e conduzir à hospitalização, objetivando, assim, a redução da morbidade da doença.

Lins MGM, Motta MEFA, Silva GAP. Risk factors to persistent diarrhea in infants. Arq Gastroenterol 2003;40(4):239-246.

ABSTRACT - Background - Persistent diarrhea is a multicausal disease. The analysis of risk factors for persistent diarrhea includes environmental and biological variables as well as therapeutical management. Aim - To identify risk factors for persistent diarrhea among children hospitalized with acute diarrhea. Patient and Methods - This is a case-control study. The sample consisted of 212 infants under 24 months, hospitalized with acute diarrhea, at the "Instituto Materno-Infantil de Pernambuco", Recife, PE, Brazil. Cases were infants with persistent diarrhea and controls those with acute diarrhea. Cases and controls were compared to a series of socio-economic, biological and clinical variables, previous morbidities and therapeutic management prior to hospital admission. Unadjusted and adjusted odds ratio were used with the respective $95 \%$ confidence intervals. It was adopted the level of significance of $5 \%$. Logistic regression analysis was conducted to control for potential confounding factors. Results - The risk of persistent diarrhea was higher for infants with: dysentery, fever at the onset of diarrhea, fasting and taking antibiotics prior to hospital admission. The variables that showed the highest adjusted odds ratios for persistent diarrhea were infants living in households without refrigerator and perianal hyperemia at hospital admission. Conclusions - The improvement of environmental conditions and an adequate clinical management of diarrhea for hospitalized infants may contribute to the reduction of diarrhea morbidity.

HEADINGS - Diarrhea, infantile. Risk factors. Hyperemia.

\section{REFERÊNCIAS BIBLIOGRÁFICAS}

1. Alam S, Malik MA. Outcomes of cases of persistent diarrhea after discharge. Indian J Pediatr 2000;67:34-6.

2. Andrade JA, Gomes TA, Fagundes-Neto U. Letalidade em lactentes com diarréia persistente: fatores de risco associados ao óbito. Arq Gastroenterol 1998;35:62-8

3. Barros FC, Victora CG. Epidemiologia da saúde infantil: um manual para diagnósticos comunitários. São Paulo: Hucitec-UNICEF; 1991. 176 p.

4. Bhan MK, Bhandari N, Bhatnagar S, Bahl R. Epidemiology \& management of persistent diarrhoea in children of developing countries. Indian J Med Res 1996;104:103-14.

5. Bhandari N, Bahl R, Saxena M, Taneja S, Bhan MK. Prognostic factors for persistent diarrhoea managed in a community setting. Indian J Pediatr 2000;67:739-45

6. Bhatnagar S, Bhan MK, Singh KD, Shrivastav R. Prognostic factors in hospitalized children with persistent diarrhea: implications for diet therapy. J Pediatr Gastroenterol Nutr 1996;23:151-8.

7. Brasil. Ministério da Saúde. Assistência e controle das doenças diarréicas. Brasília; 1993

8. Cama RI, Parashar UD, Taylor DN, Hickey T, Figueroa D, Ortega YR, Romero S, Perez J, Sterling CR, Gentsch JR, Gilmas RH, Glass RI. Enteropathogens and other factors associated with severe disease in children with acute watery diarrhea in Lima, Peru. J Infect Dis 1999;179:1139-44.

9. Fagundes-Neto U, Scaletsky ICA. The gut at war: the consequences of enteropathogenic Escherichia coli infection as a factor of diarrhea and malnutrition. São Paulo Med J 2000;118:21-9.

10. Feachem RG. Interventions for the control of diarrhoeal disease among young children: promotion of personal and domestic hygiene. Bull World Health Organ 1984:62:467-76.

11. Feachem RG, Koblinsky MA. Interventions for the control of diarrhoeal disease among young children: promotion of breast-feeding. Bull World Health Organ 1984;62:271-91.

12. Fontaine O. Oral rehydration therapy: a critical component in integrated management of childhood illness. J Pediatr Gastroenterol Nutr 2000;30:490-5.

13. Fuchs SC, Victora CG, Fachel J. Modelo hierarquizado: uma proposta de modelagem aplicada à investigação de fatores de risco para diarréia grave. Rev Saúde Pública 1996;30:168-78.

14. Gibson RS. Principles of nutritional assessment. New York: Oxford University; 1990. p.187-93.

15. Gonsales Yucra O, Aguilar Liendo AM, Levine W, Tamayo Meneses L, Hannover E, Zamora Gutierrez A, Revollo R, Rios JL, Damiani E, Espada A, Ramirez S, Rocha E. Caracteristicas de niños con diarrea persistente en la consulta ambulatorial del Hospital del Niño Dr. Ovidio Aliaga Uría. Rev Soc Boliv Pediatr 1999;38:94-9.
16. Gracey M. Nutritional effects and management of diarrhoea in infancy. Acta Paediatr 1999;88 Suppl:110-26.

17. Guandalini S. Prolonged diarrhea: etiology and pathogenesis. In: Gracey M, Walker-Smith JA, editors. Diarrheal disease. Philadelphia: Lippincott-Raven; 1977. (Nestlé nutrition workshop series, v. 38). p.153-70.

18. Guandalini S. Treatment of acute diarrhea in the new millennium. J Pediatr Gastroenterol Nutr 2000;30:486-9.

19. Hoque BA, Chakraborty J, Chowdhury JT, Chowdhury UK, Ali M, el Arifeen S, Sack RB. Effects of environmental factors on child survival in Bangladesh: a case-control study. Public Heatlh 1999;113:57-64.

20. Karim AS, Akhter S, Rahman MA, Nazir MF. Risk factors of persistent diarrhea in children below five years age. Indian J Gastroenterol 2001;20:59-61.

21. Ketema 1, Lulseged S. Persistent diarrhhoea: socio-demographic and clinical profile of 264 children seen at a referral hospital in Addis Ababa. Ethiop Med J 1997;35:161-8.

22. Lima AA, Moore SR, Barboza MS, Soares AM, Schleupner MA, Newman RD, Sears CL, Nataro JP, Fedorko DP, Wuhib T, Schorling JB, Guerrant RL. Persistent diarrhea signals a critical period of increased diarrhea burdens and nutritional shortfalls: a prospective cohort study among children in northeastern Brazil. J Infect Dis 2000;181:1643-51.

23. Mitra AK, Rahman MM, Fuchs GJ. Risk factors and gender differentials for death among children hospitalized with diarrhoea in Bangladesh. J Health Popul Nutr 2000;18:151-6.

24. Monteiro CA, Zuniga HP, Benício MH, Szarfarc SC. Estudo das condições de saúde das crianças do município de São Paulo, SP (Brasil), 1984-1985. I Aspectos Metodológicos, características sócio-econômicas e ambiente físico. Rev Saúde Pública 1986;20:435-45.

25. Oo KN, Aung WW, Thida M, Toe MM, Lwin HH, Khin EE. Relationship of breast-feeding and hand-washing with dehydration in infants with diarrhoea due to Escherichia coli. J Health Popul Nutr 2000;18:93-6.

26. Pinto EA, Barros-Filho AA, Barros MB. Fatores de risco para diarréia persistente em crianças hospitalizadas. Arq Gastroenterol 1998;35:126-31.

27. Sodeinde O, Adeyemo AA, Gbadegesin RA, Ademowo OG. Persistent diarrhoea in Nigerian children aged less than five years: a hospital-based study. J Diarrhoeal Dis Res 1997; 15:155-60.

28. Sondemann M, Jakobsen MS, Molbak K, Martins C, Aaby P. Episode-specific risk factors for progression of acute diarrhoea to persistent diarrhoea in west African children. Trans R Soc Trop Med Hyg 1999;93:65-8.

29. Taniguchi K, Rikimaru T, Yartey JE, Akpedonu P, Armar-Klemesu MA, Nkrumah FK, Kamiya H, Kishi K, Armar DA. Immunological background in children with persistent diarrhea in Ghana. Pediatr Int 1999;41:162-7.

30. Teklemariam S, Getaneh T, Bekele F. Environmenthal determinants of diarrhoea morbidity in under-five children, Keffa-Sheka zone, south west Ethiopia. Ethiop Med J 2000;38:27-34. 
31. Vella V, Tomkins A, Borghesi A, Migliori GB, Adriko BC, Crevatin E. Determinants of child nutrition and mortality in north-west Uganda. WHO Bull 1992;70:637-43

32. Victora CG, Huttly SR, Fuchs SC, Olinto MT. The role of conceptual frameworks in epidemiological analysis: a hierarchical approach. Int J Epidemiol 1997;26:224-7.
33. Wierzba TF, El-Yazeed RA, Savarino SJ, Mourad AS, Rao M, Baddour M, ElDeen NA, Naficy AB, Clemens JD. The interrelationship of malnutrition and diarrhea in a periurban area outside Alexandria, Egypt. J Pediatr Gastroenterol Nutr 2001;32:189-96.

Recebido em 11/9/2002. Aprovado em 17/2/2003. 